\title{
Palinomorfos do perfil sedimentar de uma turfeira em São Francisco de Paula, Planalto Leste do Rio Grande do Sul, Sul do Brasil ${ }^{1}$
}

\author{
ADRIANA LEONHARDT ${ }^{2,3}$ e MARIA LUISA LORSCHEITTER ${ }^{2}$
}

(recebido: 2 de fevereiro de 2006; aceito: 30 de setembro de 2006)

\begin{abstract}
Palynomorphs of the sedimentary profile of a bog, São Francisco de Paula, Eastern Plateau of Rio Grande do Sul, southern Brazil). This paper aims at giving basic reference material to the study of plant succession in the Quaternary Brazilian southern plateau. Therefore, it presents the palynological analysis of 22 samples, taken along one sedimentary profile of $286 \mathrm{~cm}$, in a bog of Rio Grande do Sul Eastern Plateau, corresponding approximately to the last 25000 years. The chemical processing of the samples followed the conventional method and the analysis was made by light microscopy. Palynomorphs corresponding to 10 fungi, 6 algae, 3 bryophytes and 16 pteridophytes were examined. The material, especially spores, is described and illustrated. The descriptions are accompanied, whenever possible, by ecological data of the original organism.
\end{abstract}

Key words - cryptogams, palynology, Quaternary, southern Brazil, taxonomy

RESUMO - (Palinomorfos do perfil sedimentar de uma turfeira em São Francisco de Paula, Planalto Leste do Rio Grande do Sul, Sul do Brasil). O trabalho tem como objetivo fornecer material de referência básico para o estudo de sucessão vegetal no Quaternário do Planalto Sul-brasileiro. Para tanto, apresenta a palinologia de 22 amostras, retiradas ao longo de um perfil sedimentar de $286 \mathrm{~cm}$, em uma turfeira do Planalto Leste do Rio Grande do Sul, correspondendo aproximadamente aos últimos 25.000 anos. O processamento químico das amostras seguiu o método convencional e a análise foi feita em microscopia fotônica. Foram examinados palinomorfos correspondentes a 10 fungos, 6 algas, 3 briófitos e 16 pteridófitos. O material, especialmente esporos, é descrito e ilustrado. As descrições são acompanhadas, sempre que possível, de dados ecológicos do organismo de origem.

Palavras-chave - criptógamos, palinologia, Quaternário, Sul do Brasil, taxonomia

\section{Introdução}

O estudo de sucessão vegetal permite o conhecimento dos processos envolvidos na gênese das formações vegetais e, assim, a melhor compreensão da dinâmica e das tendências naturais da vegetação e clima, importantes em monitoramento ambiental. Como pólen, esporos e outros palinomorfos refletem na morfologia a espécie de origem, a palinologia de perfis sedimentares, aliada a datações radiométricas, fornece uma das principais ferramentas a este estudo. Para tanto, a prévia identificação dos palinomorfos contidos nos sedimentos é necessária, o que torna de grande valia os catálogos palinológicos de referência, tanto da flora atual quanto do material sedimentar.

A dinâmica da mata com Araucária no Holoceno e a gênese das turfeiras do Planalto do Rio Grande do Sul tem despertado a atenção de muitos pesquisadores,

\footnotetext{
1. Parte da dissertação de mestrado da primeira autora, Programa de Pós-graduação em Botânica, Universidade Federal do Rio Grande do Sul.

2. Universidade Federal do Rio Grande do Sul, Instituto de Biociências, Departamento de Botânica. Av. Bento Gonçalves 9500, 91540-000 Porto Alegre, RS, Brasil.

3. Autor para correspondência: adriana.leonhardt@yahoo.com.br
}

preocupados com a preservação desses ecossistemas, mas ainda são escassos os trabalhos sobre palinologia de sedimentos da região, envolvendo sucessão vegetal (Roth \& Lorscheitter 1993, Behling et al. 2001, 2004).

Visando contribuir para o desenvolvimento dos estudos de sucessão vegetal no Planalto Leste do Sul do Brasil, é apresentada aqui a primeira parte da análise palinológica qualitativa de um perfil sedimentar, em uma turfeira atual do Município de São Francisco de Paula, abrangendo palinologia de fungos, algas, briófitos e pteridófitos como material de referência. $\mathrm{O}$ espectro polínico corresponde ao encontrado num intervalo de $286 \mathrm{~cm}$ de comprimento, envolvendo 22 amostras, distribuídas ao longo do perfil. A base da seqüência foi datada em 24.930 \pm 180 anos AP (Antes do Presente), Beta 194784. O intervalo corresponde, portanto, ao final do Pleistoceno e a todo o Holoceno. O trabalho apresenta caracterizações sucintas de cada material, com dados ecológicos do organismo de origem (sempre que possível) e fotomicrografias ópticas.

\section{Material e métodos}

A turfeira estudada localiza-se em Alpes de São Francisco (29²9' S e 50³7’ W), Município de São Francisco de Paula (figura 1). O perfil foi coletado aproximadamente no 


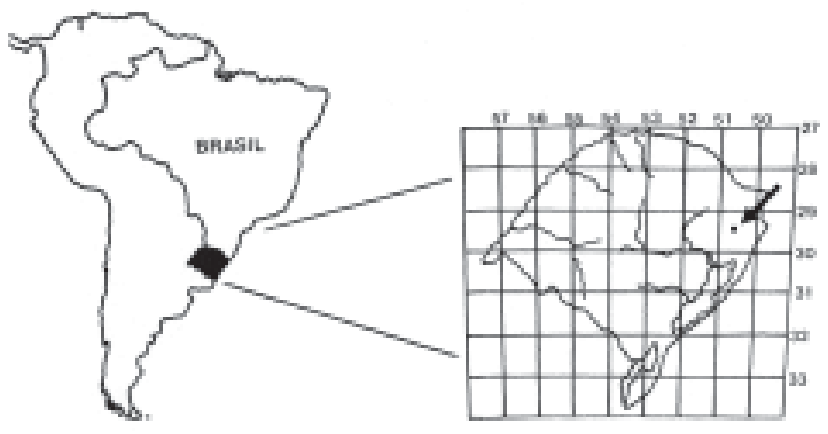

Figura 1. Estado do Rio Grande do Sul e a turfeira estudada a 29²9’ S e 50³7’ W (•), Município de São Francisco de Paula, Planalto Leste.

Figure 1. Rio Grande do Sul State and the studied bog at 29²9' S and 50³7'W (•), São Francisco de Paula Municipality, eastern Plateau.

centro da turfeira, correspondendo à porção mais espessa do pacote sedimentar. Para a coleta foi usado o Amostrador de Hiller (Faegri \& Iversen 1989), em 11 secções de $26 \mathrm{~cm}$, que foram posteriormente alinhadas, formando um perfil sedimentar de $286 \mathrm{~cm}$ de comprimento. Ao longo desse perfil foram retiradas 22 amostras em intervalos regulares, cada uma contendo $8 \mathrm{~cm}^{3}$ de sedimento fresco. Da base do perfil foi coletada uma amostra para datação por ${ }^{14} \mathrm{C}$, realizada no laboratório Beta Analytic Inc., Miami, Flórida. O tratamento químico das amostras para análise palinológica seguiu o método padrão (Faegri \& Iversen 1989), usando-se HCl, HF, $\mathrm{KOH}$ e acetólise, com filtragem em malha de $250 \mu \mathrm{m}$. As lâminas de microscopia foram montadas em gelatina-glicerinada e a análise das mesmas foi realizada em fotomicroscópio óptico Diaplan Leitz em aumento de 1.000 x. A palinoteca de plantas atuais do Laboratório de Palinologia do Departamento de Botânica da Universidade Federal do Rio Grande do Sul foi usada como auxiliar na identificação do material. Procurou-se sempre alcançar o nível hierárquico mais baixo possível. Nos grupos taxonômicos onde a morfologia dos esporos é muito semelhante dentro de um gênero ou família, optou-se por usar a denominação "tipo" antes do nome, conforme o usual em palinologia de sedimentos (Berglund 1986). Quando a identificação não foi possível, o material recebeu uma denominação apenas morfológica, dando possibilidade à futura identificação.

As descrições dos palinomorfos foram feitas de modo sucinto, apresentando as características básicas para a identificação do material. As medidas foram realizadas em ocular de fio móvel e convertidas para micrometros. Nas medidas dos esporos de briófitos e pteridófitos foram usados o eixo polar e o eixo equatorial. Sempre que possível foram incluídos dados ecológicos do organismo de origem, de acordo com a literatura, visando informações para análises de sucessão vegetal e de paleoambientes. A maioria das fotomicrografias foi realizada em aumento de $1.000 \mathrm{x}$ (filme T-MAX 100).

\section{Resultados e Discussão}

\author{
Fungos \\ Filo Glomeromycota \\ Classe Glomeromycetes \\ Ordem Glomerales \\ Família Glomeraceae
}

1. Glomus Tus. \& C. Tus.

Figura 2

Clamidósporo elipsoidal a esferoidal, psilado, preso a filamento de hifa cenocítica. Diâmetro: $27 \mu \mathrm{m}$. Referência: Schüßler et al. (2001). Obs: citado também para o Rio Grande do Sul pelos antigos nomes de Rhizophagites Rosendahl (Lorscheitter 1989) e Rhizophagus Dang (Neves \& Lorscheitter 1992, Neves \& Bauermann 2003). Dados ecológicos: mais comum dentre os fungos micorrízicos arbusculares. Ocorre no solo, como endossimbionte em raízes de numerosas plantas vasculares (Schüßler et al. 2001).

\author{
Filo Ascomycota \\ Classe Ascomycetes \\ Ordem Sordariales \\ Família Sordariaceae
}

\section{Gelasinospora adjuncta Cain}

Figuras 3-4

Ascósporo elipsoidal, asseptado, escuro, psilado. Superfície com pequenas perfurações circulares, densamente distribuídas. Eixo maior: 21-35 $\mu \mathrm{m}$. Eixo menor: 15-24 $\mu \mathrm{m}$. Referência: Hooghiemstra (1984). Dados ecológicos: espécie decompositora de excrementos, madeira carbonizada e lignina (Van Geel 1978).

\section{Ordem Magnaporthales Família Magnaporthaceae}

\section{Tipo Gaeumannomyces cf. caricis J. Walker} Figura 5

Esporângio discóide, escuro, psilado. Margem irregularmente lobada, com tênue poro central. Eixo maior: $22 \mu \mathrm{m}$. Eixo menor: $17 \mu \mathrm{m}$. Referência: Hooghiemstra (1984). Obs: o material é semelhante ao que Van Geel (1978) descreveu como cf. Entophlyctis lobata Willoughby \& Townley (tipo 13). Dados ecológicos: G. caricis ocorre como parasita ou saprófito, em caules e raízes de Poaceae (Von Arx 1974). 
Filo Basidiomycota

Classe Basidiomycetes

Ordem Aphyllophorales

Família Corticiaceae

\section{Tipo Athelia Persoon}

Figura 6

Células esferoidais, pequenas, escuras e psiladas, agrupadas de modo aproximadamente elipsoidal. Diâmetro das células: 8-15 $\mu \mathrm{m}$. Agrupamento: eixo maior 36-62 $\mu \mathrm{m}$, eixo menor 25-52 $\mu \mathrm{m}$. Referência: Hooghiemstra (1984). Dados ecológicos: o gênero inclui possivelmente patógenos de liquens e algas (Kirk et al. 2001).

\section{Classe Ustomycetes \\ Ordem Ustilaginales \\ Família Tilletiaceae}

\section{Tilletia sphagni Naw}

Figuras 7-9

Esporo aproximadamente esferoidal, frágil e hialino, psilado. Superfície com placas poligonais, dando ao grão um aspecto facetado. Pequeno. Diâmetro do esporo: 11-13 $\mu \mathrm{m}$. Diâmetro das placas: cerca de $4 \mu \mathrm{m}$. Referências: Matteri (1987), Van Geel (1978). Dados ecológicos: parasita de esporângios de várias espécies de Sphagnum, inibindo a formação dos esporos do musgo (Matteri 1987).

Outros esporos

\section{Tipo Spirotremesporites multiplex Dueñas Figuras 10-12}

Esporo fusiforme, escuro, psilado. Fendas paralelas e helicoidais (em geral quatro) em torno do maior eixo. Eixo maior: $52 \mu \mathrm{m}$. Eixo menor: $15 \mu \mathrm{m}$. Referência: Dueñas (1979). Obs: Incertae sedis, possível afinidade com Xylariaceae (Dueñas 1979). Primeira citação para o Quaternário do Rio Grande do Sul.

\section{Esporo 1}

Figuras 13-14

Esferoidal, pequeno, escuro, microequinado. Espinhos muito finos, diminutos, densamente dispostos. Perfuração evidente, com espessamento marginal. Pequeno. Diâmetro do grão: $10 \mu \mathrm{m}$.

\section{Esporo 2 \\ Figura 15}

Fusiforme, hialino, estriado. Estrias longitudinais, aproximadamente paralelas. Fragmento de hifa às vezes presente em uma das extremidades. Pequeno. Eixo maior: 17-18 $\mu \mathrm{m}$. Eixo menor: 9-11 $\mu \mathrm{m}$.

9. Esporo 3

Figura 16

Alongado, extremidades levemente truncadas, com característico espessamento mais escuro. Psilado. Fendas longitudinais muito finas e paralelas. Pequeno. Eixo maior: 13-16 $\mu \mathrm{m}$. Eixo menor: $7 \mu \mathrm{m}$.

\section{Esporo 4}

Figuras 17-18

Tétrade tetragonal. Esporo escuro, microequinado. Espinhos irregularmente dispersos, mais concentrados na porção marginal junto a dois esporos. Pequeno. Diâmetro da tétrade: $15 \mu \mathrm{m}$. Diâmetro do esporo: $8 \mu \mathrm{m}$.

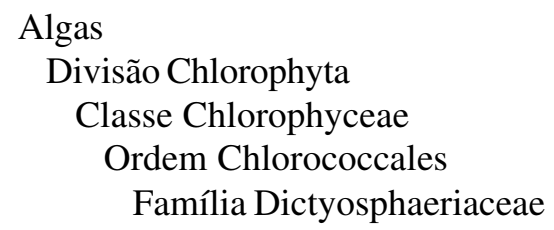

11. Botryococcus Kützing

Figura 19

Colônia irregularmente lobada, de tamanho variável, escura e psilada, com muitos indivíduos unicelulares, densa e concentricamente dispostos. Diâmetro da colônia: 28-35 $\mu \mathrm{m}$. Referência: Hooghiemstra (1984). Dados ecológicos: em água doce, em poças ou lagos (Erdtman 1969).

$$
\begin{aligned}
& \text { Classe Zygnemaphyceae } \\
& \text { Ordem Zygnematales } \\
& \text { Família Zygnemataceae }
\end{aligned}
$$

\section{Debarya (De Bary) Wittrock}

Figuras 20-22

Zigósporo formado por dois hemisférios complementares, radialmente simétricos. Hemisférios com um anel característico em vista frontal, com projeções estriadas-ruguladas no centro e estrias paralelas, radialmente dispostas, na periferia. Cada hemisfério com pólo truncado em vista lateral. Diâmetro equatorial: 32-46 $\mu \mathrm{m}$. Altura de cada hemisfério: $22 \mu \mathrm{m}$. Referência: Hooghiemstra (1984). Obs: os zigósporos encontrados apresentam-se com apenas um dos hemisférios. Dados ecológicos: em água doce, estagnada e com pouca profundidade (Van Geel \& Van Der Hammen 1977).

13. Mougeotia C. A. Agardh

Figura 23 
Zigósporo quadrangular a levemente retangular em vista frontal, hialino, psilado, com pequenas dobras características nos ângulos. Eixo maior: 30-50 $\mu$ m. Eixo menor: 22-46 $\mu \mathrm{m}$. Referências: Van Geel (1978), Hooghiemstra (1984). Dados ecológicos: em água doce e solos úmidos (Joly 2002).

\section{Spirogyra Link}

Figuras 24-26

Zigósporo esferoidal a elipsoidal, hialino ou amarelado, reticulado. Retículo muito grosseiro. Eixo maior: 60-135 $\mu \mathrm{m}$. Referência: Hooghiemstra (1984). Dados ecológicos: como em Mougeotia.

\section{Zygnema C. A. Agardh}

Figuras 27-29

Zigósporo elipsoidal, hialino, psilado, apresentando reentrâncias circulares características, pequenas ou robustas, regularmente distribuídas por toda a superfície. Eixo maior do zigósporo: 43-50 $\mu \mathrm{m}$. Eixo menor: $27 \mu \mathrm{m}$. Diâmetro das reentrâncias: 2-8 $\mu \mathrm{m}$. Referências: Van Geel (1978), Hooghiemstra (1984). Dados ecológicos: em água doce e solos úmidos (Smith 1987, Joly 2002).

\section{Incertae sedis}

16. Pseudoschizaea rubina Rossignol ex Christopher Figura 30-33

Grão circular em vista frontal e elíptico em vista lateral, hialino e estriado. Estrias finas e concêntricas em vista frontal, formando anéis e espiras às vezes incompletos. Finas estrias paralelas em vista lateral. Diâmetro em vista frontal: 28-43 $\mu \mathrm{m}$. Referência: Christopher (1976). Obs: o material é citado em trabalhos antigos como a forma A de Concentricystes rubinus Rossignol. Dados ecológicos: grãos sem afinidade botânica confirmada, possivelmente originados de algas dulciaqüícolas (Rossignol 1962).

\section{Briófitos \\ Divisão Anthocerotophyta \\ Classe Anthocerotopsida \\ Ordem Anthocerotales \\ Família Anthocerotaceae}

\section{Anthoceros punctatus L.}

\section{Figura 34-35}

Radiossimétrico, heteropolar, circular em vista polar. Trilete, raios finos com extremidade bifurcada. Reticulado-equinado no pólo distal, psilado no proximal. Retículo grosseiro, com espinhos alongados e freqüentemente bifurcados a trifurcados, alguns simples, sobre o muro. Eixo equatorial: 55-66 $\mu \mathrm{m}$. Dados ecológicos: anual, em campos e pântanos sombreados (Menéndez 1962).

\section{Phaeoceros laevis (L.) Prosk.} Figura 36-38

Radiossimétrico, heteropolar, circular a subtriangular em vista polar. Trilete, raios finos com extremidade bifurcada. Microequinado a psilado no pólo distal e, em geral, psilado no proximal. Espinhos finos, escassos a abundantes, regular a irregularmente distribuídos. Eixo equatorial: 62-74 $\mu \mathrm{m}$. Dados ecológicos: sobre solos úmidos, em locais abrigados. Comum em margem de arroios, rios, vertentes e em campos úmidos (Menéndez 1962).

$$
\begin{aligned}
& \text { Divisão Bryophyta } \\
& \text { Classe Sphagnopsida } \\
& \text { Ordem Sphagnales } \\
& \text { Família Sphagnaceae }
\end{aligned}
$$

19. Tipo Sphagnum recurvum P. Beauv. Figuras 39-40

Radiossimétrico, heteropolar, subtriangular em vista polar, com zonas interangulares levemente convexas. Trilete. Espessamento trilobado característico no pólo distal, lobos irregularmente crenulados. Psilado no pólo proximal. Eixo equatorial: $28-48 \mu \mathrm{m}$. Obs: a espécie ocorre na atual turfeira, segundo a morfologia descrita por Yano et al. (1985). Dados ecológicos: as espécies de Sphagnum são de solos ácidos, sujeitos a inundações, como brejos e pântanos, em especial turfeiras. Também em lagoas rasas, em densas e extensas colônias (Joly 2002). Primeira citação da espécie para o Quaternário do Rio Grande do Sul.

Pteridófitos

$$
\begin{aligned}
& \text { Divisão Pteridophyta } \\
& \text { Classe Filicopsida } \\
& \text { Subclasse Polypodiidae } \\
& \text { Ordem Polypodiales } \\
& \text { Subordem Polypodiineae } \\
& \text { Família Hymenophyllaceae }
\end{aligned}
$$

\section{Tipo Hymenophyllum Sm.}

Figuras 41-42

Radiossimétrico, heteropolar e hialino, subtriangular a circular em vista polar. Trilete. Micropapilado. Papilas muito finas, densamente distribuídas por toda a superfície. Eixo equatorial: 38-47 $\mu \mathrm{m}$. Dados ecológicos: na América tropical o gênero ocorre especialmente em florestas nebulares e florestas úmidas de montanha. É 

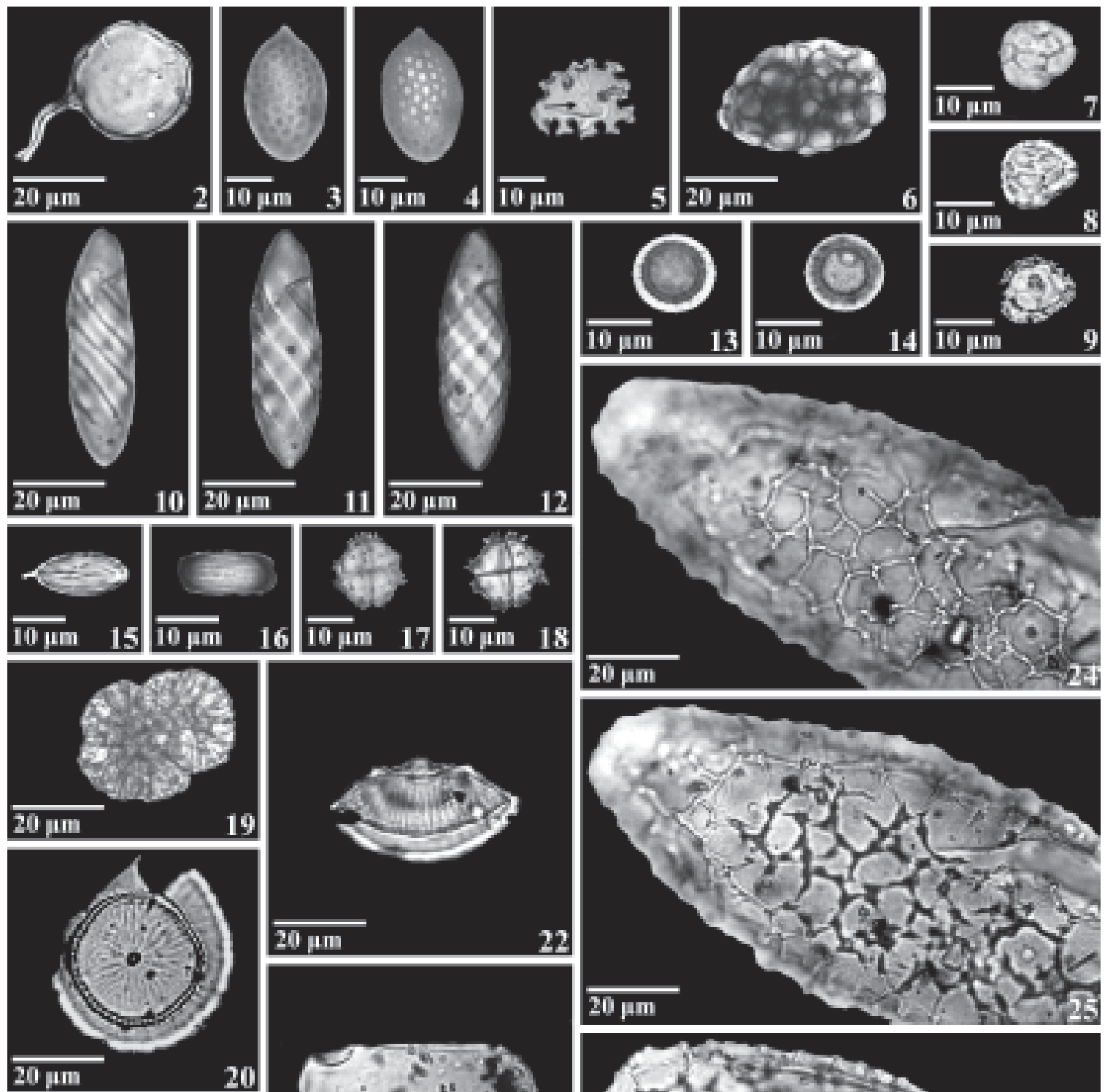

19
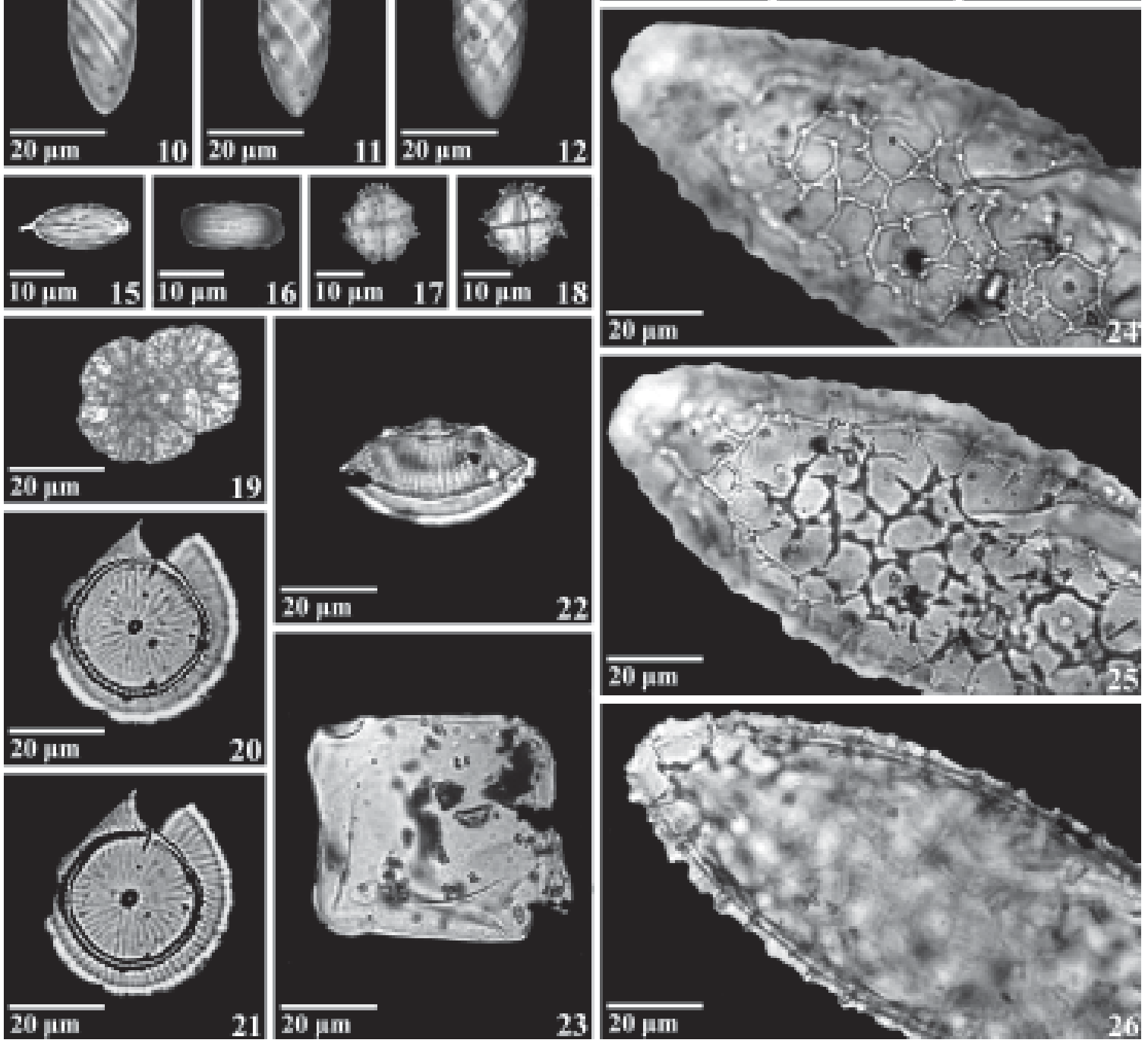
23

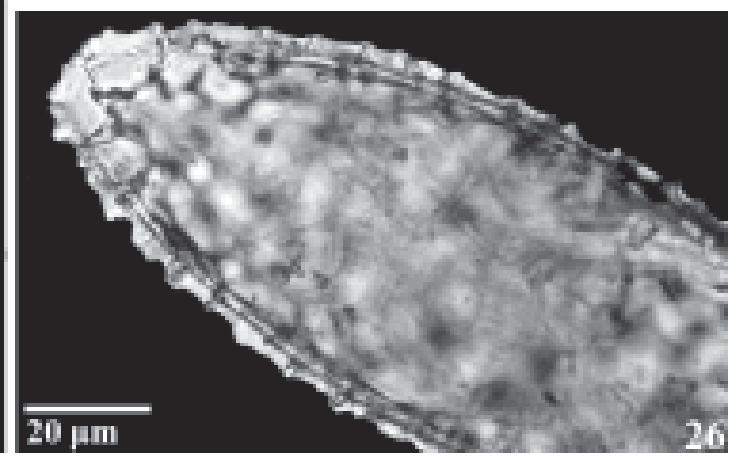

Figuras 2-26. Palinomorfos. 2. Glomus. 3-4.Gelasinospora adjuncta: $1^{\circ}-2^{\circ}$ pl. 5. Tipo Gaeumannomyces cf. caricis. Tênue poro central (seta). 6. Tipo Athelia. 7-9. Tilletia sphagni: $1^{\circ}-3^{\circ} \mathrm{pl}$. 10-12. Tipo Spirotremesporites multiplex: $1^{\circ}-3^{\circ}$ pl. $13-14$. Esporo 1: $1^{\circ}-2^{\circ} \mathrm{pl}$. 15. Esporo 2. 16. Esporo 3. 17-18. Esporo 4: $1^{\circ}-2^{\circ} \mathrm{pl}$. 19. Botryococcus. 20-22. Debarya. 20-21. Vista frontal: $1^{\circ}-2^{\circ} \mathrm{pl}$. 22. Vista lateral. 23. Mougeotia. 24-26. Spirogyra: $1^{\circ}-3^{\circ} \mathrm{pl}$. $(\mathrm{pl}=\mathrm{planos})$.

Figures 2-26. Palynomorphs. 2. Glomus. 3-4. Gelasinospora adjuncta: $1^{\mathrm{st}}-2^{\text {nd }} \mathrm{pl}$. 5. Gaeumannomyces cf. caricis type. Tenuous central pore (arrow). 6. Athelia type. 7-9. Tilletia sphagni: $1^{\text {st }}-3^{\text {rd }} \mathrm{pl}$. 10-12. Spirotremesporites multiplex type: $1^{\text {st }} 3^{\text {rd }} \mathrm{pl}$. $13-14$. Spore 1: $1^{\text {st }}-2^{\text {nd }}$ pl. 15. Spore 2. 16. Spore 3. 17-18. Spore 4: $1^{\text {st }}-2^{\text {nd }}$ pl. 19. Botryococcus. 20-22. Debarya. 20-21. Frontal view: $1^{\text {st }-2} 2^{\text {nd }}$ pl. 22. Lateral view. 23. Mougeotia. 24-26. Spirogyra: $1^{\text {st }}-3^{\text {rd }} \mathrm{pl}$. ( $\mathrm{pl}=$ planes $)$. 

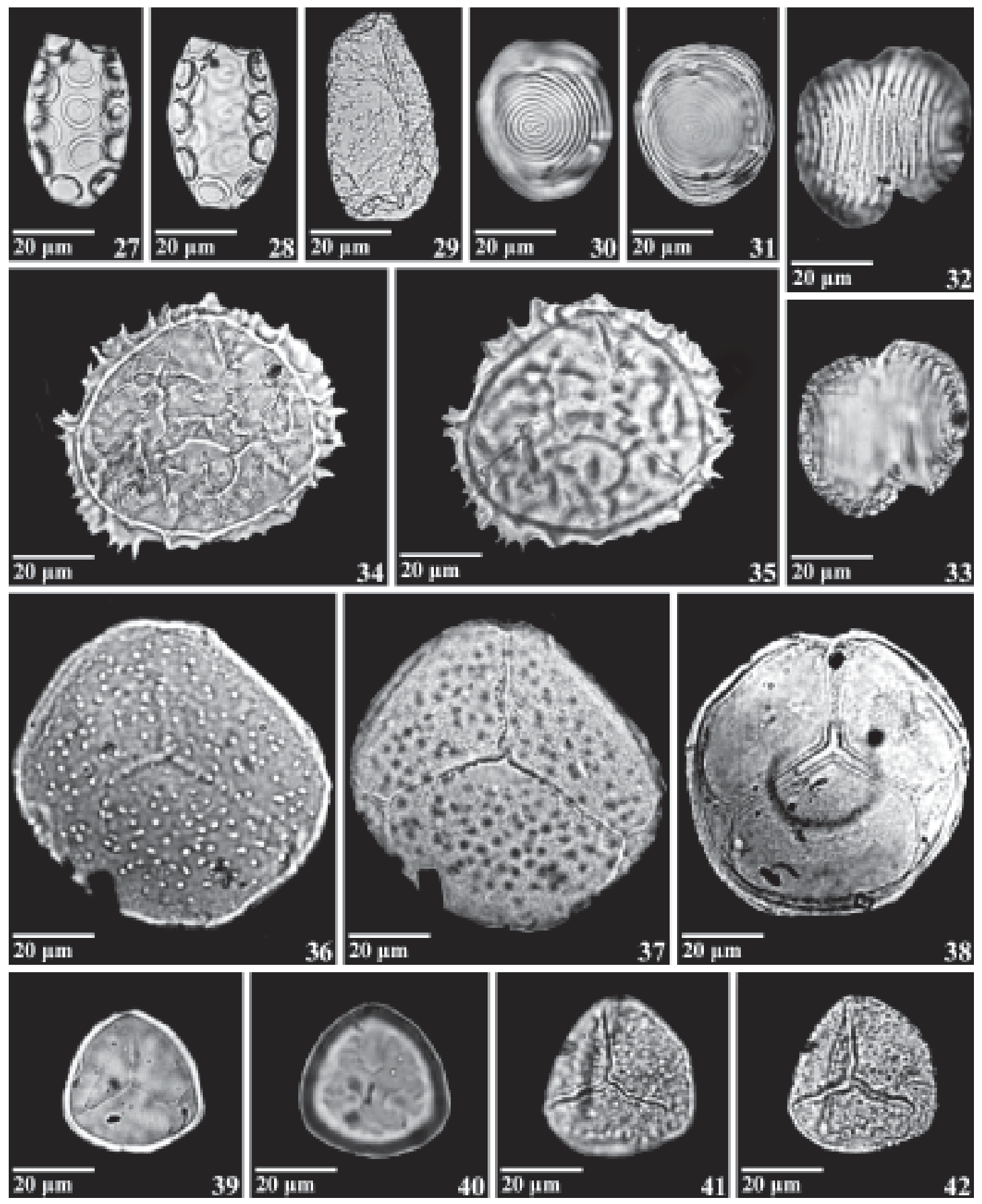

Figuras 27-42. Palinomorfos. 27-29. Zygnema. 27-28: $1^{\circ}-2^{\circ}$ pl. 29. Reentrâncias menores. 30-33. Pseudoschizaea rubina. $30-31$. Vista frontal: $1^{\circ}-2^{\circ} \mathrm{pl} .32-33$. Vista lateral (fragmentado): $1^{\circ}-2^{\circ} \mathrm{pl} .34-35$. Anthoceros punctatus (PD): $1^{\circ}-2^{\circ}$ pl. 36-38. Phaeoceros laevis. 36-37 (PD), equinado (raios vistos por transparência): $1^{\circ}-2^{\circ} \mathrm{pl}$. 38. (PP), psilado. 39-40. Tipo Sphagnum recurvum (PP): $1^{\circ}-2^{\circ} \mathrm{pl}$ (lobos vistos por transparência). 41-42. Tipo Hymenophyllum (PP): $1^{\circ}-2^{\circ} \mathrm{pl} .(\mathrm{pl}=$ planos; $\mathrm{PD}=$ pólo distal; $\mathrm{PP}=$ pólo proximal).

Figures 27-42. Palynomorphs. 27-29. Zygnema. 27-28: $1^{\text {st }-2 \text { nd }}$ pl. 29. Smaller reentrances. 30-33. Pseudoschizaea rubina. $30-31$. Frontal view: $1^{\text {st }}-2^{\text {nd }} \mathrm{pl}$. 32-33. Lateral view (fragmented): $1^{\text {st }}-2^{\text {nd }}$ pl. 34-35. Anthoceros punctatus $(\mathrm{PD}): 1^{\text {st }}-2^{\text {nd }} \mathrm{pl}$. 36-38. Phaeoceros laevis. 36-37 (PD), echinate (laesura seen due to transparency): $1^{\text {st }}-2^{\text {nd }} \mathrm{pl} .38$. (PP), psilate. 39-40. Sphagnum recurvum type (PP): $1^{\text {st }}-2^{\text {nd }} \mathrm{pl}$ (lobes seen due to transparency). 41-42. Hymenophyllum type $(\mathrm{PP}): 1^{\text {st }}-2^{\text {nd }} \mathrm{pl} .(\mathrm{pl}=$ planes $; \mathrm{PD}=$ distal pole $; \mathrm{PP}=$ proximal pole). 
menos freqüente em florestas tropicais de mais baixas altitudes. Muitas espécies são epifíticas ou epipétricas, em florestas úmidas (Tryon \& Tryon 1982). Comuns do Planalto do Rio Grande do Sul (Lorscheitter et al. 1999).

Família Osmundaceae

\section{Osmunda L.}

Figuras 43-44

Radiossimétrico, heteropolar, circular em vista polar. Trilete, raios longos e finos. Verrucado, verrugas de tamanho, forma e distribuição irregulares. Exospório fino, facilmente dobrável, tornando o grão frágil. Robusto. Eixo equatorial: 71-82 $\mu \mathrm{m}$. Obs: freqüentemente encontrado em fragmentos nas amostras. Dados ecológicos: em ambientes úmidos, raro em terrenos com boa drenagem. Mais freqüente em locais abertos, mas também nos sombreados. $\mathrm{Na}$ América tropical geralmente ocorre em ambientes sempre úmidos, abertos ou não, como pântanos, turfeiras, campos e margens de lagos (Tryon \& Tryon 1982). Comuns no Planalto do Rio Grande do Sul (Lorscheitter et al. 1998).

\section{Família Dicksoniaceae}

\section{Dicksonia sellowiana Hook.} Figura 45

Radiossimétrico, heteropolar, subtriangular a triangular em vista polar, ângulos truncados onde o exospório é nitidamente mais espesso. Trilete, margo robusto. Espessamentos largos e irregulares no pólo distal, psilado no proximal. Robusto. Eixo equatorial: 65-80 $\mu \mathrm{m}$. Dados ecológicos: arborescente, geralmente em matas úmidas de montanha e florestas nebulares na América tropical, na base de encostas ou ao longo de córregos (Tryon \& Tryon 1982). No Rio Grande do Sul é encontrada em altitudes de 60 a $1.200 \mathrm{~m}$, sendo freqüente na Mata com Araucária (Lorscheitter et al. 1999).

Família Cyatheaceae

\section{Cyatheaceae}

Figura 46

Radiossimétrico, heteropolar, subtriangular a triangular em vista polar. Trilete, margo robusto. Psilado. Exospório com espessamento uniforme. Eixo equatorial: 46-54 $\mu \mathrm{m}$. Dados ecológicos: em florestas tropicais de montanha, florestas úmidas de montanha ou em florestas nebulares (Tryon \& Tryon 1982, Lorscheitter et al. 1999).

\section{Família Pteridaceae}

\section{Pteris L.}

Figuras 47-49

Radiossimétrico, heteropolar, subtriangular a triangular em vista polar, cingulado. Trilete. Irregularmente verrucado-rugulado no pólo distal, psilado no proximal. Eixo equatorial: $52 \mu \mathrm{m}$. Dados ecológicos: na América tropical cresce em florestas úmidas, na borda de clareiras e em capoeiras, em florestas nebulares ou de galeria, ocasionalmente sobre rochedos. Também em locais alterados (Tryon \& Tryon 1982). No Rio Grande do Sul, a maioria das espécies é terrícola, em florestas (Lorscheitter et al. 2001).

\section{Família Blechnaceae}

25. Blechnum cf. imperiale (Fee \& Glaziou) H. Chr. Figura 50

Bilateral, heteropolar, oblato. Elíptico em vista polar e plano-convexo em vista equatorial. Monolete. Psilado, amarelo escuro. Robusto. Eixo polar: 55-68 $\mu \mathrm{m}$. Eixo equatorial maior: 74-93 $\mu \mathrm{m}$. Dados ecológicos: espécie vastamente dispersa em locais pantanosos e úmidos, junto de águas correntes no campo, mas também em locais mais secos em matas e capoeiras. No Rio Grande do Sul ocorre preferencialmente em turfeiras do Planalto (Sehnem 1968).

\section{Tipo Blechnum L.}

Figura 51

Bilateral, heteropolar, oblato. Elíptico em vista polar e plano-convexo em vista equatorial. Monolete. Psilado, amarelo claro. Eixo polar: 30-43 $\mu \mathrm{m}$. Eixo equatorial maior: $52-65 \mu \mathrm{m}$. Obs: as diferenças entre os esporos do Tipo Blechnum e $B$. cf. imperiale são o tamanho e a coloração. Dados ecológicos: gênero de plantas terrícolas, rupestres, algumas vezes epifíticas. $\mathrm{Na}$ América tropical cresce em florestas tropicais, florestas de montanha e florestas nebulares, e ao longo de córregos e rios. Também ocorre em pântanos e turfeiras, em florestas de galeria e em savanas. Algumas espécies vivem em locais alterados (Tryon \& Tryon 1982). Gênero predominante no hemisfério sul (Sehnem 1968).

\section{Família Polypodiaceae}

27. Tipo Microgramma vacciniifolia (Langsd. \& Fisch.) Copel.

Figuras 52-54

Bilateral, heteropolar, oblato. Elíptico em vista polar e plano-convexo em vista equatorial. Monolete. 
Verrucado, verrugas irregulares, distribuídas por toda a superfície, diminuindo em direção ao pólo proximal. Eixo polar: $40 \mu \mathrm{m}$. Eixo equatorial: $65 \mu \mathrm{m}$. Dados ecológicos: a espécie é epifítica a epipétrica, em locais sombreados e abertos, desde florestas de baixas altitudes até florestas nebulares (Lorscheitter et al. 2005).

\section{Tipo Polypodium L. \\ Figuras 55-56}

Bilateral, heteropolar, oblato a suboblato. Elíptico em vista polar, plano-convexo em vista equatorial. Monolete. Verrucado, verrugas uniformemente distribuídas. Eixo polar: 43-50 $\mu \mathrm{m}$. Eixo equatorial maior: 57-67 $\mu \mathrm{m}$. Obs: diferencia-se do Tipo M. vacciniifolia por apresentar verrugas uniformes por toda a superfície. Dados ecológicos: em nichos variados. Na América tropical as espécies do gênero geralmente crescem em áreas baixas de florestas tropicais, florestas de montanha ou florestas nebulares. (Tryon \& Tryon 1982). No Rio Grande do Sul ocorrem, em geral, como epifíticas em matas (Lorscheitter et al. 2005).

29. Tipo Pleopeltis angusta Humb. \& Bonpl. ex Willd. Figuras 57-59

Bilateral, heteropolar, oblato. Elíptico em vista polar, plano-convexo em vista equatorial. Monolete. Verrucado, verrugas muito pequenas, distribuídas densamente por toda a superfície. Perfurações finas e esparsas, entre as verrugas. Robusto. Eixo polar: $49 \mu \mathrm{m}$. Eixo equatorial maior: $76 \mu \mathrm{m}$. Dados ecológicos: a espécie é epifítica, em locais ao nível do mar até altitude de $1.000 \mathrm{~m}$, em matas nebulares de montanha. Também comum em florestas decíduas (Lorscheitter et al. 2005). Primeira citação para o Quaternário do Rio Grande do Sul.

\section{Classe Lycopodiopsida \\ Ordem Lycopodiales \\ Família Lycopodiaceae}

\section{Huperzia Bernh.}

Figuras 60-61

Radiossimétrico, heteropolar, subtriangular em vista polar. Trilete. Foveolado no pólo distal, psilado no proximal. Eixo equatorial: 46-53 $\mu \mathrm{m}$. Dados ecológicos: gênero com a maioria das espécies epífíticas, ocorrendo dentro de matas (Lorscheitter et al. 1998).

\section{Lycopodiella alopecuroides (L.) Cranfill Figuras 62-64}

Radiossimétrico, heteropolar, subtriangular a circular em vista polar, levemente cingulado. Trilete. Rugulado- reticulado no pólo distal, com projeções grosseiras e pequeno espessamento aproximadamente quadrangular central. Pólo proximal tuberculado entre os raios. Eixo equatorial: $60 \mu \mathrm{m}$. Dados ecológicos: terrícola, geralmente em turfeiras e campos úmidos (Lorscheitter et al. 1998).

\section{Tipo Lycopodiella cernua (L.) Pic. Serm.} Figuras 65-66

Radiossimétrico, heteropolar, subtriangular em vista polar. Trilete. Rugulado no pólo distal, psilado no proximal. Eixo equatorial: $43 \mu \mathrm{m}$. Dados ecológicos: a espécie é terrícola, em margem de estradas (Lorscheitter et al. 1998).

\section{Tipo Lycopodium clavatum $\mathrm{L}$.}

Figuras 67-69

Radiossimétrico, heteropolar, subtriangular em vista polar. Trilete. Reticulado, retículo grosseiro no pólo distal, diminuindo em direção ao pólo proximal, onde a superfície torna-se psilada. Eixo equatorial: $43 \mu \mathrm{m}$. Obs: diferencia-se dos esporos de L. clavatum adicionados às amostras para cálculo de concentração polínica por apresentar coloração bem mais clara. Grãos raramente encontrados. Dados ecológicos: espécie geralmente em capoeiras e ambientes semi-abertos em florestas nebulares (Tryon \& Tryon 1982, Lorscheitter et al. 1998).

Ordem Selaginellales

Família Selaginellaceae

37. Selaginella marginata (Humb. \& Bonpl. ex Willd.) Spring

Figuras 70-72

Radiossimétrico, heteropolar, subtriangular a circular em vista polar. Trilete. Baculado, báculas pequenas, menores no pólo proximal. Eixo equatorial: 32-38 $\mu \mathrm{m}$. Comprimento das báculas: cerca de $3 \mu \mathrm{m}$. Dados ecológicos: terrícola, em ambientes úmidos, comuns no Rio Grande do Sul (Lorscheitter et al. 1998).

Ordem Isoetales

Família Isoetaceae

38. Isoetes $\mathrm{L}$.

Micrósporo

Figura 73

Bilateral, heteropolar, peroblato a oblato, hialino. Elíptico em vista polar, plano-convexo em vista equatorial. Monolete. Psilado. Paraexospório psilado a escabrado, envolvendo frouxamente o grão. Eixo polar, 

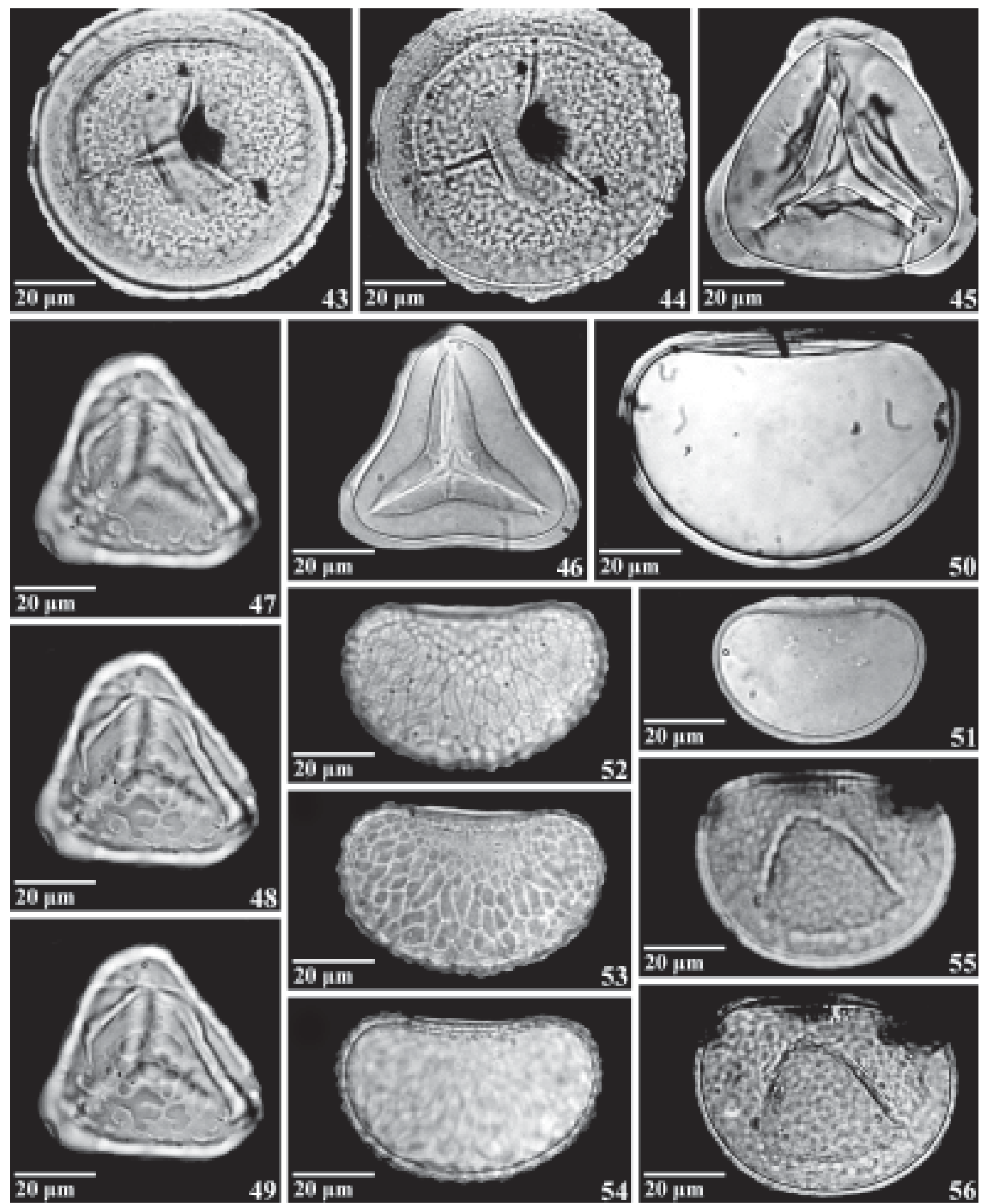

Figuras 43-56. Palinomorfos. 43-44. Osmunda (PP): $1^{\circ}-2^{\circ}$ pl. 45. Dicksonia sellowiana (PP). 46. Cyatheaceae (PP). 47-49. Pteris (PD): $1^{\circ}-3^{\circ} \mathrm{pl}$. 50. Blechnum cf. imperiale (EQ). 51. Tipo Blechnum (EQ). 52-54. Tipo Microgramma vacciniifolia (EQ): $1^{\circ}-3^{\circ} \mathrm{pl}$. 55-56. Tipo Polypodium (EQ): $1^{\circ}-2^{\circ} \mathrm{pl}$. ( $\mathrm{pl}=$ planos; $\mathrm{PD}=$ pólo distal; $\mathrm{PP}=$ pólo proximal; $\mathrm{EQ}=$ vista equatorial).

Figure 43-56. Palynomorphs. 43-44. Osmunda (PP): $1^{\text {st }-2^{\text {nd }}}$ pl. 45. Dicksonia sellowiana (PP). 46. Cyatheaceae (PP). 47-49. Pteris (PD): $1^{\text {st }}-3^{\text {rd }} \mathrm{pl} .50$. Blechnum $\mathrm{cf}$. imperiale (EQ). 51. Blechnum type (EQ). 52-54. Microgramma vacciniifolia type (EQ): $1^{\text {st }}-3^{\text {rd }}$ pl. 55-56. Polypodium type (EQ): $1^{\text {st }-2^{\text {nd }}} \mathrm{pl}$. $(\mathrm{pl}=$ planes; $\mathrm{PD}=$ distal pole; $\mathrm{PP}=$ proximal pole; $\mathrm{EQ}=$ equatorial view). 

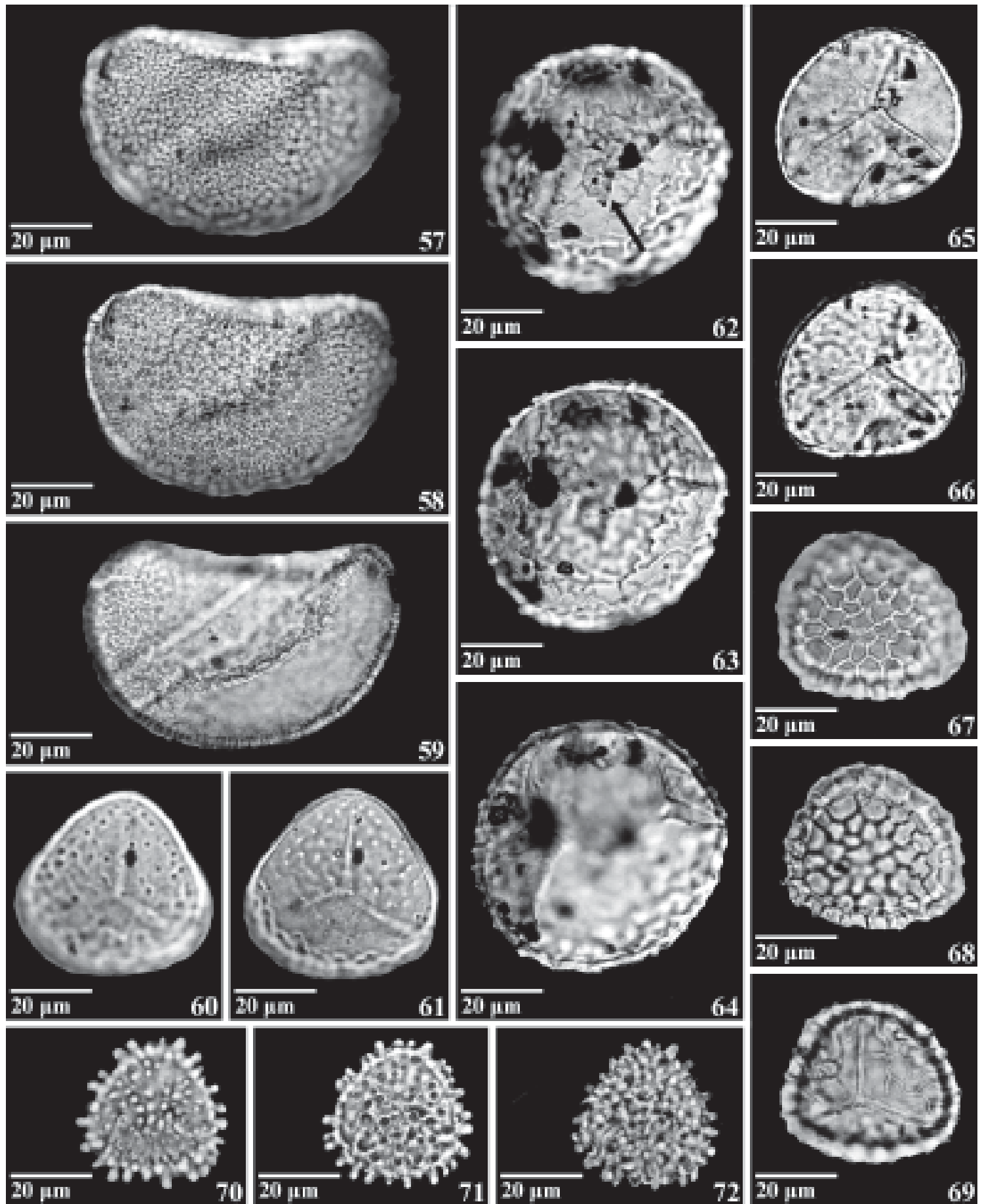

66

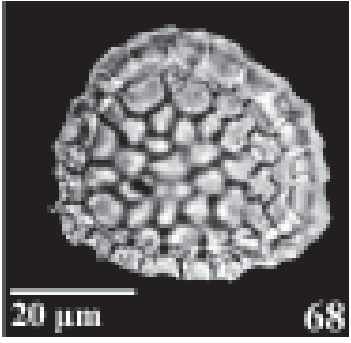

4

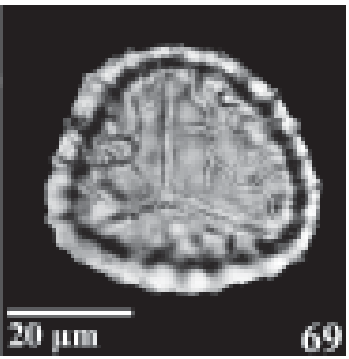

Figuras 57-72. Palinomorfos. 57-59. Tipo Pleopeltis angusta (EQ): $1^{\circ}-3^{\circ} \mathrm{pl}$. 60-61. Huperzia (PD): $1^{\circ}-2^{\circ}$ pl. 62-64. Lycopodiella alopecuroides (PD): $1^{\circ}-3^{\circ} \mathrm{pl}$. 62. Espessamento central (seta). 65-66. Tipo Lycopodiella cernua (PP): $1^{\circ}-2^{\circ} \mathrm{pl}$. 67-69. Tipo Lycopodium clavatum $(\mathrm{PD}): 1^{\circ}-3^{\circ} \mathrm{pl} .70-72$. Selaginella marginata $(\mathrm{PD}): 1^{\circ}-3^{\circ} \mathrm{pl} .(\mathrm{pl}=$ planos; $\mathrm{PD}=$ pólo distal; $\mathrm{PP}=$ pólo proximal; $\mathrm{EQ}=$ vista equatorial).

Figures 57-72. Palynomorphs. 57-59. Pleopeltis angusta type (EQ): $1^{\text {st }-3^{\text {rd }}}$ pl. 60-61. Huperzia (PD): $1^{\text {st }-2 ~}{ }^{\text {nd }}$ pl. 62-64. Lycopodiella alopecuroides (PD): $1^{\text {st }} 3^{\text {rd }} \mathrm{pl}$. 62. Central thick part (arrow). 65-66. Lycopodiella cernua type (PP): $1^{\text {st }-2^{\text {nd }}} \mathrm{pl}$. 67-69. Lycopodium clavatum type $(\mathrm{PD}): 1^{\mathrm{st}}-3^{\text {rd }} \mathrm{pl}$. 70-72. Selaginella marginata $(\mathrm{PD}): 1^{\text {st }}-3^{\text {rd }} \mathrm{pl}$. $(\mathrm{pl}=$ planes; $\mathrm{PD}=$ distal pole; $\mathrm{PP}=$ proximal pole; $\mathrm{EQ}=$ equatorial view). 
com paraexospório: 22-30 $\mu \mathrm{m}$, sem paraexospório: 14-15 $\mu \mathrm{m}$. Eixo equatorial maior, com paraexospório: 32-37 $\mu \mathrm{m}$, sem paraexospório: 26-31 $\mu \mathrm{m}$.

\section{Megásporo}

Figuras 74-80
Tetraédrico-globoso, radiossimétrico, heteropolar. Subtriangular a circular em vista polar, com cingulum. Trilete de raios muito longos, em geral com extremidade distintamente auriculada. Reticulado a escabrado no pólo distal, psilado no proximal. Exospório externo lamelar e lacunoso, lacuna fina a espessa. Eixo polar: 595-740 $\mu \mathrm{m}$.
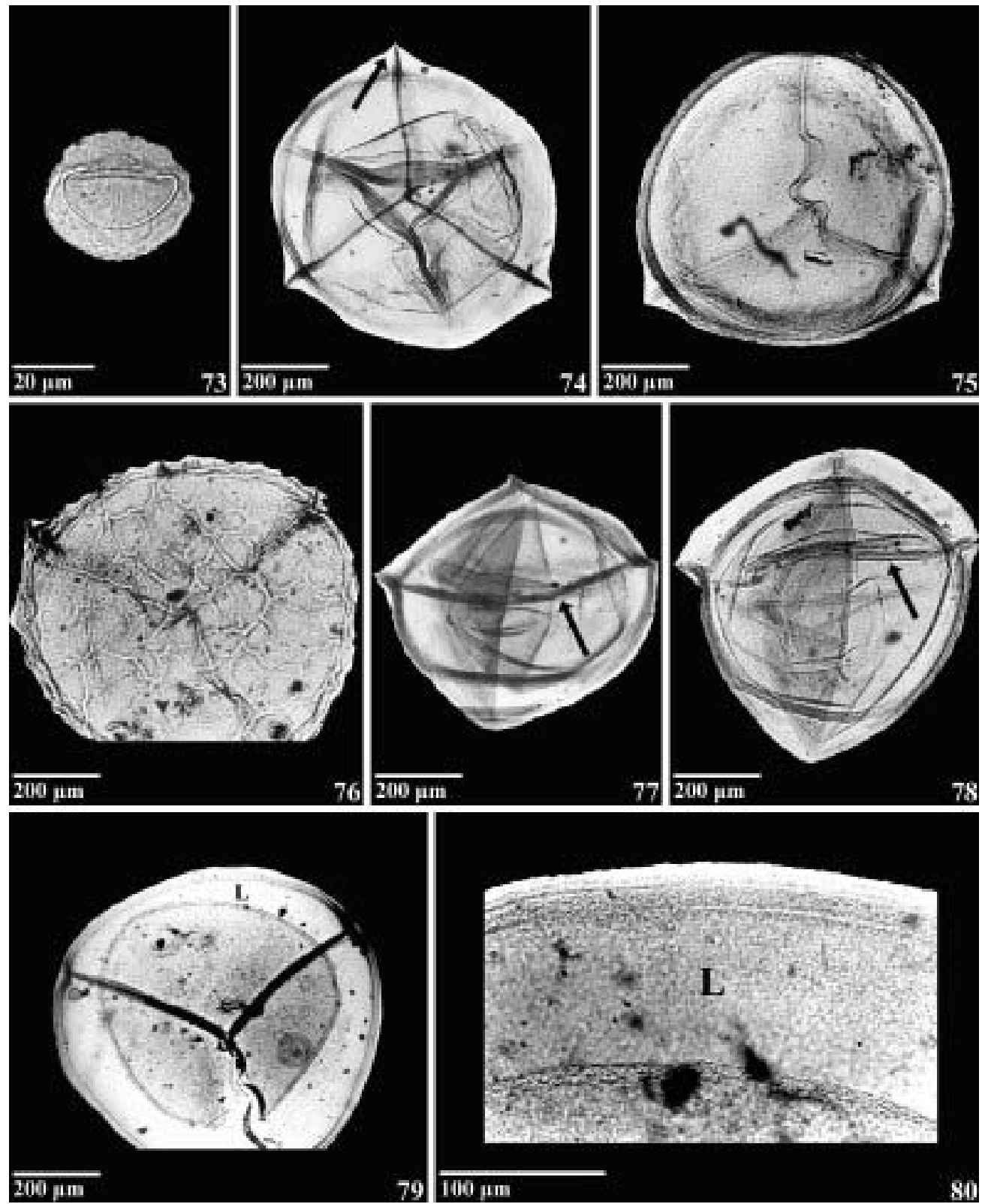

Figuras 73-80. Palinomorfos. 73. Isoetes, micrósporo (EQ), paraexospório desenvolvido. 74-80. Isoetes, megásporos. 74 (PP), psilado, raios com aurículas evidentes (seta). 75 (PD), escabrado (marca trilete vista por transparência). 76 (PP), reticulado (retículo visto por transparência). 77-78 (EQ), cingulum evidente (seta). 79 (PP), exospório externo com lacuna espessa (L). 80. Detalhe do exospório lamelar com lacuna desenvolvida $(\mathrm{L}) .(\mathrm{PD}=$ pólo distal; $\mathrm{PP}=$ pólo proximal; $\mathrm{EQ}=$ vista equatorial $)$.

Figures 73-80. Palynomorphs. 73. Isoetes, microspore (EQ), developed paraexospore. 74-80. Isoetes, megaspores. 74 (PP), psilate, evident auriculate arms (arrow). 75 (PD), scabrate (laesura seen due to transparency). 76 (PP), reticulate (reticulum seen due to transparency). 77-78 (EQ), evident cingulum (arrow). 79 (PP), outer exospore, with thick lacuna (L). 80. Detail of the lamellar exospore, with developed lacuna $(\mathrm{L}) .(\mathrm{PD}=$ distal pole; $\mathrm{PP}=$ proximal pole; $\mathrm{EQ}=$ equatorial view $)$. 
Eixo equatorial: 660-880 $\mu \mathrm{m}$. Obs: os megásporos foram encontrados no material retido na malha de $250 \mu \mathrm{m}$, durante a filtragem das amostras. Dados ecológicos: em lagos, preferencialmente de águas rasas, poças ou córregos, solos arenosos úmidos e em turfeiras (Tryon \& Tryon 1982, Lorscheitter 1998). Primeira citação de megásporos de Isoetes para o Quaternário do Rio Grande do Sul.

A análise palinológica qualitativa permitiu encontrar 35 palinomorfos distintos ( 10 fungos, 6 algas, 3 briófitos e 16 pteridófitos) preservados ao longo do perfil sedimentar, que abrange o final do último estágio glacial pleistocênico e todo o Holoceno. Desse total, quatro são citações novas para o Quaternário do Rio Grande do Sul: Tipo Spirotremesporites multiplex, Tipo Sphagnum recurvum, Tipo Pleopeltis angusta e Tipo Lycopodiella cernua, além de megásporos de Isoetes. A grande diversidade de palinomorfos encontrados nas amostras indica o alto potencial da palinologia de perfis sedimentares de turfeiras no estudo da dinâmica da vegetação e clima dos últimos milênios no Planalto do Sul do Brasil.

Agradecimentos - as autoras agradecem ao CNPq e à Capes pelos auxílios financeiros e bolsas concedidas, que tornaram possível a realização deste trabalho.

\section{Referências bibliográficas}

BEHLING, H., BAUERMANN, S.G. \& NEVES, P.C.P. 2001. Holocene environmental changes in São Francisco de Paula region, southern Brazil. Journal of South American Earth Sciences 14:631-639.

BEHLING, H., PILLAR, V.D.P., ORLÓCI, L. \& BAUERMANN, S.G. 2004. Late Quaternary Araucaria forest, grassland ("campos"), fire and climate dynamics, studied by high resolution pollen, charcoal and multivariate analysis of the Cambará do Sul core in southern Brazil. Palaeogeography, Palaeoclimatology, Palaeoecology 203:277-297.

BERGLUND, B.E. 1986. Handbook of Holocene Palaeoecology and Palaeohydrology. John Wiley \& Sons, New York.

CHRISTOPHER, R.A. 1976. Morphology and taxonomy status of Pseudoschizea Thiergart and Frantz ex R. Potonié emend. Micropaleontology 22:143-150.

DUEÑAS, H. 1979. Estudio palinologico de los $35 \mathrm{mts}$. superiores de la seccion Tarragona, Sabana de Bogota. Caldasia 12:539-571.

ERDTMAN, G. 1969. Handbook of Palynology. Hafner Publishing Co., New York.
FAEGRI, K. \& IVERSEN, J. 1989. Textbook of pollen analysis. $4^{\text {th }}$ ed., John Wiley \& Sons, New York.

HOOGHIEMSTRA, H. 1984. Vegetational and climatic history of the High Plain of Bogotá, Colombia: A continuos record of the last 3,5 million years. Strauss \& Cramer, Amsterdam.

JOLY, A.B. 2002. Botânica: introdução à taxonomia vegetal. $13^{\mathrm{a}}$ ed., Editora Nacional, São Paulo.

KIRK, P.M., CANNON, P.F., DAVID, J.C. \& STALPERS, J.A. 2001. Dictionary of the fungi. $9^{\text {th }}$ ed., CAB Internacional, Cambridge.

LORSCHEITTER, M.L. 1989. Palinologia de sedimentos quaternários do testemunho T15, Cone de Rio Grande, Atlântico Sul, Brasil. Descrições taxonômicas, parte II. Pesquisas 22:89-127.

LORSCHEITTER, M.L., ASHRAF, A.R., BUENO, R.M. \& MOSBRUGGER, V. 1998. Pteridophyte of Rio Grande do Sul flora, Brazil. Part I. Palaeontographica 246:1-113.

LORSCHEITTER, M.L., ASHRAF, A.R., WINDISCH, P.G. \& MOSBRUGGER, V. 1999. Pteridophyte of Rio Grande do Sul flora, Brazil. Part II. Palaeontographica 251:71-235.

LORSCHEITTER, M.L., ASHRAF, A.R., WINDISCH, P.G. \& MOSBRUGGER, V. 2001. Pteridophyte of Rio Grande do Sul flora, Brazil. Part III. Palaeontographica 260:1-165.

LORSCHEITTER, M.L., ASHRAF, A.R., WINDISCH, P.G. \& MOSBRUGGER, V. 2005. Pteridophyte of Rio Grande do Sul flora, Brazil. Part V. Palaeontographica 270:1-180.

MATTERI, C.M. 1987. Esporas de hongos em capsulas de Sphagnum magellanicum de Tierra del Fuego. Boletin de la Associación Latinoamericana de Paleobotánica y Palinologia 10:6.

MENÉNDEZ, G.G.H. 1962. Estudio de las Anthocerotales y Marchantiales de la Argentina. Universidad Nacional de Tucumán, Instituto Miguel Lillo, Tucumán.

NEVES, P.C.P. \& BAUERMANN, S.G. 2003. Catálogo palinológico de coberturas quaternárias do estado do Rio Grande do Sul (Guaíba e Capão do Leão), Brasil. Descrições taxonômicas, parte I: fungos, algas, palinomorfos outros e fragmentos de invertebrados. Pesquisas, Botânica 53:121-149.

NEVES, P.C.P. \& LORSCHEITTER, M.L. 1992. Palinologia de sedimentos de uma mata tropical paludosa em Terra de Areia, Planície Costeira Norte, Rio Grande do Sul, Brasil. Descrições taxonômicas, parte I: fungos, algas, briófitos, pteridófitos, palinomorfos outros e fragmentos de invertebrados. Acta Geologica Leopoldensia 15:83-114.

ROSSIGNOL, M. 1962. Analyse pollinique de sediments marins quaternaries em Israel. II Sédiments Pleistocenes. Pollen et Spores 4:121-148.

ROTH, L. \& LORSCHEITTER, M.L. 1993. Palynology of a bog in Parque Nacional de Aparados da Serra, East Plateau of Rio Grande do Sul, Brazil. Quaternary of South America and Antarctic Peninsula 8:39-69.

SCHÜßLER, A., SCHWARZOTT, D. \& WALKER, C. 2001. A new fungal phylum, the Glomeromycota: phylogeny and evolution. Mycological Research 105:1413-1421. 
SEHNEM, A. 1968. Blecnáceas. In Flora ilustrada catarinense (R. Reitz, ed.). Herbário Barbosa Rodrigues, Itajaí, p.1-90.

SMITH, G.M. 1987. Botanica criptogamica. $4^{\mathrm{a}}$ ed., v.1. Fundação Calouste Gulbenkian, Lisboa.

TRYON, R.M. \& TRYON, A.F. 1982. Ferns and allied plants. Springer-Verlag, New York.

VAN GEEL, B. 1978. A paleoecological study of Holocene peat bog section in Germany and the Netherlands. Review of Palaeobotany and Palynology 25:1-120.
VAN GEEL, B. \& VAN DER HAMMEN, T. 1977. Zygnemataceae in Quaternary Colombian sediments. Review of Palaeobotany and Palynology 25:377-392.

VONARX, J.A. 1974. The genera of fungi sporulating in pure cultura, A.R. Gantner Verlag, Vaduz.

YANO, O., PIRANI, J.R. \& SANTOS, D.P. 1985. O gênero Sphagnum (Bryopsida) nas regiões Sul e Sudeste do Brasil. Revista Brasileira de Botânica 8:55-80. 